\title{
¿Es rentable el periodismo de calidad? Disposición al pago en Brasil y España
}

\author{
Is quality journalism profitable? Intent \\ to pay in Brazil and Spain
}

\author{
Eduardo Francisco RODRÍGUEZ GÓMEZ1 \\ Claudia FAVARETTO LOUZADA²
}

\section{Resumen}

La profesión periodística afronta una crisis que, al cambio de siglo, se ha agravado con el establecimiento del paradigma digital y son muchos, medios y periodistas, los que se han visto incapacitados para adaptarse al nuevo entorno y aceptar la reforma transversal que la red ha incitado en el modelo de negocio. Este artículo explora la predisposición al pago por el periodismo digital de calidad con la intención de aportar ideas a los profesionales del sector y orientarles en su búsqueda para un negocio sostenible. El texto presenta las reflexiones de 26 periodistas y académicos de Brasil y España que se han recogido mediante entrevistas estandarizadas no programadas. Los resultados muestran la necesidad de fidelizar al público mediante contenido digital exclusivo, participativo y de fácil acceso, aunque se evidencian igualmente las carencias en la formación y adaptación de los profesionales, así como las limitaciones impuestas por el mercado, los internautas y las herramientas digitales.

Palabras clave: Calidad periodística. Empresa mediática. Pago. Periodismo digital. Usuarios de Internet.

\begin{abstract}
Journalism is facing an acute crisis that, with the turn of the century, has aggravated due to the establishment of the digital paradigm. Many media and journalists have been unable to adapt themselves to the new environment and have not yet accepted the cross-sectional reform that the Internet has provoked in the business model. This paper explores the intent to pay for quality digital journalism with the aim of providing a guiding groundwork for professionals that would shed light in their search for a viable business. The text presents the reflections made by 26 journalists and academics from Brazil and Spain whose opinions were collected through semi-structured interviews. The results not only show the need to maintain the audience's loyalty with unique, participatory and accessible digital content, but also reveal the gaps in the training and adaptation of professionals as well as the limitations imposed by the market, Internet users and social digital tools.
\end{abstract}

Keywords: Quality journalism. Media company. Payment. Digital journalism. Internet users.

\section{Introducción}

La profesión periodística se encuentra actualmente en un contexto comprometido que ha propiciado la precariedad laboral, los despidos masivos y los cierres de grandes y pequeños medios que antaño formaron parte de nuestro imaginario cultural. En este entorno cambiante, que aún sufre de cierto rechazo

\footnotetext{
1 Universidad Carlos III de Madrid, Departamento de Periodismo y Comunicación Audiovisual. C/ Madrid, 126, Edificio 17, 28903, Getafe, Madrid, España. Dirección de correspondencia/Correspondence to: E.F. RODRÍGUEZ GÓMEZ. E-mail: <edrodrig@hum.uc3m.es>.

2 Universidad Autónoma de Barcelona, Departamento de Periodismo y Ciencias de la Comunicación, Programa Oficial de Doctorado en Comunicación y Periodismo. Bellaterra, Barcelona, España.

Recebido el 15/1/2016 y aceptado para su publicación el 16/6/2016.
} 
profesional, dominar las nuevas herramientas, generar especialidades y aprender a trabajar con diferentes velocidades son algunas de las inquietudes ordinarias que los profesionales tienen hoy en día; por encima de todo ello, la principal preocupación es afrontar la dominante condición del 'todo gratis' que Internet ha impuesto. "Tenemos que querer más a la profesión, somos muy corporativos y a veces nos defendemos poco. Por primera vez en muchos años, estamos unidos para parar la sangría económica" (RIVERO, 2014, p.19).

Los académicos y expertos que se han interesado por la intención de la audiencia en la compra de contenidos informativos no han explorado hasta el momento las posibilidades económicas que pudiera brindar un periodismo digital de calidad. Estos agentes han analizado sustancialmente los contenidos de la prensa digital - en ocasiones de la televisión y nunca de la radio -, como consecuencia de la disminución de los lectores y la inversión publicitaria.

Hay cuatro grupos diferenciados. Por un lado, se encuentran los expertos que aportan sus reflexiones basadas en los conocimientos y experiencias personales que han adquirido a lo largo de su desarrollo profesional, siendo su aportación de utilidad como marco de contexto para posteriores estudios. Mientras los más críticos, como Brader (2006) y McChesney y Pickard (2011), auguran un entorno opresivo donde la población se encuentra incapacitada intelectualmente para comprender la necesidad de existencia de medios independientes a los centros de poder, los más optimistas (BROCK, 2013, HUNTER; WASSENHOVE, 2010; JUSTEL, 2012;) predicen un mercado próspero sin contratiempos financieros para los medios y en el que las presiones de accionistas y anunciantes apenas son tenidas en cuenta. Entre ambas posturas, hallamos a los que se afanan en analizar las aristas de los diferentes modelos empresariales, bien sobre las características que hacen 'viral' un contenido, convirtiéndolo en potencial producto de consumo (BERGER; MILKMAN, 2012), bien sobre el mecenazgo y la aportación altruista de la audiencia (LEWIS, 2008; NELSON, 2011; REQUEJOALEMÁN; LUGO-OCANDO, 2014), bien sobre las nuevas vías de financiación del branded journalism "periodismo de marcas" (DI GIACOMO, 2013).

Por otro lado, están los estudios de mercado llevados a cabo por instituciones privadas que adoptan una postura economicista, y muestran una mayor preocupación por los resultados mercantiles y los ratings de audiencia y un menor interés por la incidencia de la calidad informativa sobre la disposición al pago de los lectores. Bajo este rubro se encuentran los estudios del American Press Institute (2015), la KPMG International (2008, 2010, 2011) y la Oriella PR Network (2011-2013); así como los trabajos de Bruno y Nielsen (2012) y las investigaciones del Instituto Reuters, llevadas a cabo por Newman et al. (2015) y Newman y Levy $(2013,2014)$. En Brasil, hallamos el estudio de la Associação Nacional de Jornais (MARSHALL, 2014) y en España, los informes de la Asociación de Editores de Diarios Españoles (2014), la Asociación para la Investigación de Medios de Comunicación $(2012,2013,2014)$ y la Asociación de la Prensa de Madrid (PALACIO, 2013, 2014).

Un tercer grupo plasma las reflexiones de expertos respecto al fenómeno del pago por contenidos. En su conjunto, muestran que no hay un modelo de negocio viable universal, ya que para cada diario, en cada país y en cada etapa se establece una miríada de peculiaridades privativas al contexto espacial y temporal en el que cada proyecto se desarrolla. Al igual que con el primer grupo, encontramos la línea optimista de Fondevila (2012), Saba (2011) y Shields (2009), quienes consideran que la rentabilidad de las nuevas empresas informativas es asumible ya que, a largo plazo, se trata de proyectos con unos costes fijos que no representan cargas insoslayables. Edmonds (2009), Hamilton (2004) y Salaverría (2012), sin embargo, se suman al carro de los pesimistas al considerar que incluso las personas más interesadas y mejor informadas no pagarán por una información que se cristalice con posterioridad en supuestos beneficios sociales. Bahón (2014) constataría la poca confianza de las instituciones y de las empresas mediáticas en la suscripción anual como una fuente de ingresos consistente.

En el último grupo ubicamos las investigaciones científicas que, en parte o en su totalidad, se centran en la disposición de la audiencia al pago por contenidos informativos - a diferencia de las obras de la primera categoría, que exhibían un acercamiento más general -. Destacan los proyectos del Pew Research Center (MITCHELL, 2014; MITCHELL; PAGE, 2015; MITCHEL; ROSENSTIEL, 2013); y las exploraciones de la profesora 
Chyi, (2012) o junto con Chyi y Lee (2012a, 2012b). En España también destacan las investigaciones lideradas por Ramírez de La Piscina et al. (2014), y por Vara-Miguel et al. (2014), así como el estudio de Goyanes (2015) sobre los medios locales. La idea principal que se destila de todos ellos es la dificultad de los medios para conseguir que los lectores paguen, siendo los jóvenes los más dispuestos pero los menos interesados en información de actualidad, aunque hay casos de éxito basados en la calidad periodística y el contenido exclusivo.

El objetivo del presente trabajo es demostrar el interés que la audiencia ha evidenciado por el periodismo de calidad (TRANSPARENCY INTERNATIONAL, 2012) y la posibilidad de materializar esa sugestión en la compra de productos y contenidos periodísticos digitales. Nuestra finalidad es, por tanto, aportar ideas a los profesionales del sectory orientarles en su búsqueda para un negocio sostenible. La hipótesis principal es: La audiencia está dispuesta a pagar por un producto de calidad que se distinga de lo que otros ofertan de manera gratuita.

En función de esta hipótesis trataremos de dar respuesta a las siguientes cuestiones:

1) ¿Los profesionales mantienen una percepción y acercamiento positivo hacia las nuevas formas de hacer periodismo?

2) ¿El entorno 2.0 ha contribuido al surgimiento de diferentes modelos de negocio sostenibles dentro del ámbito de los contenidos digitales?

3) ¿Las nuevas herramientas digitales son, en su empleo experto y razonable, perfectos complementos a la rigurosa metodología que exige el periodismo online de calidad?

4) ¿Son las redes sociales herramientas útiles para el tratamiento informativo en profundidad?

5) ¿Están los medios de comunicación convenciendo a sus públicos de la necesidad de pagar por la facilidad informativa?

6) ¿Están los periodistas haciendo llegar al lector la idea de que la calidad en la información y la autonomía del periódico dependen mucho de su audiencia?

\section{Procedimientos metodológicos}

Este artículo es resultado de la recopilación y la síntesis de las reflexiones de 26 profesionales de la comunicación, 12 de Brasil y 14 de España, obtenidas mediante entrevistas estandarizadas no programadas realizadas entre septiembre de 2014 y septiembre de 2015. El diseño de las preguntas se estableció a partir de la revisión bibliográfica que mostró las principales preocupaciones respecto al periodismo online, en particular, y de la Periodística como disciplina, en general.

La selección de expertos ha seguido las pautas queVallés (2002) señala evocando a la obra de Raymond L. Gorden:

1) ¿Quiénes tienen la información relevante?

2) ¿Quiénes son más accesibles física y socialmente? (entre los informados).

3) ¿Quiénes están más dispuestos a informar? (entre los informados y accesibles).

4) ¿Quiénes son más capaces de comunicar la información con precisión? (entre los informados, accesibles y dispuestos).

Ordenados por orden alfabético, en Brasil hemos consultado a: Benny Cohen, editor de Mídias Convergentes en Portal Uai; José Sebastião Pinheiro de Souza, editor-jefe en la Organização Jaime Câmara y en el Jornal do Tocantins; Luciamem Winck, secretaria de redacción en el Jornal Correio do Povo; Marcos Rolim, periodista y consultor en Seguridad Pública y Derechos Humanos; Natália Levien Leal, editora en el Diário Catarinense Online; Nilson Vargas, editor-jefe de Zero Hora; Raquel Vieira, editora en el Diário Catarinense; Regina Zandomenico, coordinadora del curso de Periodismo en el Centro Universitário Estácio de Sá; Silvana Araújo, coordinadora del Polo dos Cursos de Educación a Distancia de la Secretaria de Educação de Pernambuco; Valéria Miranda Kurovski, presidenta de la Fundação de Radiodifusão Educativa; Vanessa Franzosi, jefe de Reportaje en el Diário Catarinense; y A. M., que prefiere no ser identificado.

En España, hemos consultado a: Javier Chicote, redactor de ABC; Pascual Serrano, periodista e investigador en medios de comunicación; María 
José Esteso, redactora e investigadora del periódico Diagonal; Albano Dante, fundador y editor jefe de la revista Cafèambllet; Eva Belmonte, investigadora y actual responsable de proyectos en la Fundación Civio; Eric Frattini, escritor y periodista de investigación free lance; Mar Cabra, investigadora del Consorcio Internacional de Periodistas de Investigación; Daniel Montero, investigador de la revista Interviú; Esteban Urreiztieta, investigador de El Español; Miguel Jara, escritor y periodista de investigación free lance; Marcos García, investigador del Consorcio Internacional de Periodistas de Investigación; José María Irujo, director de la sección de investigación de El País; Pilar Velasco, redactora de la Cadena SER; y Montse Quesada, catedrática de periodismo de investigación.

\section{¿Qué es periodismo de calidad?}

En diferentes espacios de discusión sobre la prensa digital, editores, periodistas y académicos (DE LA TORRE; TÉRAMO, 2015; JARQUE; ALMIRÓN, 2008; LOBO, 2012; MARTíN, 2010; SOENGAS PÉREZ, 2015) coinciden en señalar que el periodismo declarativo y de presencia - que informa de lo que ocurre pero asume la propaganda institucional que se le consigna - acapara la mayor parte de los circuitos mediáticos, aportando al periodismo de calidad un halo de distinción respecto a su homónimo más popularmente extendido.

Gómez Mompart et al. (2015) parten del supuesto de que el periodismo de calidad se distingue por acceder a las fuentes primarias, apostar por el periodismo de investigación, evitar la mezcla de hechos y conjeturas y buscar la equidad entre los protagonistas de las noticias. "La información de calidad", dice José María Irujo, director de investigaciones de El País, "es sinónimo de seriedad, de rigor, de equilibrio y de otras muchas cosas, no es solo significado de denuncia y revelación de grandes escándalos".

Partimos, por tanto, de diferentes niveles cualitativos en la ejecución periodística y, siguiendo la revisión bibliográfica y las aportaciones de los expertos consultados, definimos el periodismo de calidad como aquel que:
1) Expone de forma contextualizada datos que son relevantes para la mejora social y el correcto funcionamiento de un estado democrático en un contexto local, nacional o internacional.

2) En su elaboración, existe un proceso para la verificación, ordenación y redacción de esas informaciones que es labor del/los periodista/s.

3) Es ideológicamente independiente de todo tipo de instituciones, grupos públicos y empresariales.

4) Demanda innovación, valor, eficiencia y velocidad en la manera de organizar y gestionar sus contenidos.

5) Abraza una cultura participativa, promoviendo una mayor apertura y transparencia sobre los procesos editoriales.

6) Presenta un modelo de difusión vertical donde, al mismo tiempo, productores y consumidores tienen la posibilidad de compartir la misma plataforma en un pie de igualdad.

\section{Resultados}

El periodismo tradicional afronta una crisis que, al cambio de siglo, se ha agravado con el establecimiento del paradigma digital y son muchos los periodistas que no han sabido adaptarse adecuadamente al nuevo escenario. Aun así, todos los profesionales entrevistados entienden que la digitalización ha dejado de ser una tendencia para constituirse en una realidad y que

\section{Al tiempo que se mantiene una esencia que no ha cambiado desde el origen de la profesión, debemos asimilar los nuevos aspectos para continuar siendo los protagonistas de una sociedad cada vez más necesitada de la interpretación, la elaboración y la jerarquía de la información (Nilson Vargas).}

La oferta de contenido y las posibilidades de acceso son cada vez mayores y "es preciso fidelizar al público con contenidos que sean resultado de una depuración narrativa pero que no hayan perdido su esencia ética" (José Sebastião Pinheiro de Souza). En este sentido, son visibles los avances en la cultura corporativa y su compromiso para enfocar el trabajo según las necesidades del lector, tratando de innovar en las formas de búsqueda, verificación y presentación de los textos digitales, a pesar de que en muchos casos 
"estos procesos no hayan llegado a institucionalizarse con cargos o responsables directos" (A.M.).

Desde la divulgación social y la eficaz difusión de los contenidos hasta la encriptación masiva de documentos, todos los profesionales mantienen una opinión positiva hacia las nuevas formas de realizar periodismo.

En la medida en que uno sea capaz de comprender estas tendencias, su trabajo se diferenciará cualitativamente del de otros. Aunque he de confesar que llevo varios meses haciendo noticias de 40 segundos y me siento como si estuviera en la edad de la moviola (Pilar Velasco).

Ahora, gracias a registradores.org y al Boletín Oficial del Estado, investigo a una persona sentado desde mi ordenador y en un día confecciono un informe patrimonial incontestable con toda la contratación entre instituciones públicas y privadas. Piensa que confirmar el cargo que tenía la persona ' $X$ ' hace 15 años en Telefónica es tan sencillo como hacer un click en Google, mientras que antes tenías que ir a la hemeroteca y pasarte allí una tarde (Javier Chicote).

Aunque los medios estén valorando más la estética y utilizando más la creatividad para presentar la noticia y acercarse a su target, gran parte de los encuestados coinciden en que "hay un largo camino a recorrer hasta que las publicaciones online superen la calidad de las publicaciones impresas" (Marcos Rolim). Considerando las directrices del periodismo de calidad, "la independencia de los canales, su apertura y la transparencia editorial aún siguen siendo un desafío para la mayoría" (Benny Cohen). La realidad es que "falta encontrar una fórmula que monetice el trabajo y entregue a la audiencia un periodismo que opere en profundidad" (Raquel Vieira).

Montse Quesada, entre otros, lamenta la ausencia de denuncia y de un compromiso social genuino en la labor informativa independientemente de la adjetivación que adjuntemos al concepto de periodismo - de calidad, de datos, en profundidad, etc. - "El trabajo real del periodista es conseguir el dato. Extractar la historia de una amalgama de filas y columnas ha ocurrido desde el origen de la profesión. Todo lo demás, hacerlo más vistoso y comprensible, es la carcasa" (Daniel Montero).
No se puede negar que los cambios introducidos por Internet amenazan a la sobrevivencia del diario en papel, pero desde el punto de vista de algunos expertos no se trata de una extinción del formato impreso sino de una adaptación y evolución natural. "Las estadísticas demuestran que los usuarios no ven la edición digital como una competencia, sino como una lectura complementaria" (Luciamem Winck). De hecho, hay quien observa con optimismo la consolidación de la versión impresa como la marca de apoyo a otras plataformas. "Con la presencia actuante de los medios digitales, nunca antes se había leído tanto el periódico impreso. Es sinónimo de credibilidad y esta cualidad migra con el nombre del periódico" (Vanessa Fransozi).

Todo lo relacionado con la producción del diario en papel, desde los costes hasta la publicación de las noticias, va en contra de la demanda de la gente por información en tiempo real.

Los costes de mantenimiento del modelo impreso son cada vez más altos para una respuesta cada vez más pequeña en el mercado de la publicidad (Valéria Miranda Kurovski).

Regina Zandomenico cree que es imposible competir con la inmediatez del entorno digital. Por ello, el periodismo impreso necesita encontrar otro camino, centrado en la producción de reportajes no factuales que despierten la atención del público.

En este sentido, la clave es fortalecer la marca en el entorno digital y ganar a la nueva generación de internautas (Benny Cohen).

\section{La experiencia cibersocial}

Las redes sociales han constituido una parte importante en el proceso de producción y publicación de los contenidos como canales bidireccionales. No obstante, el hecho de que la información esté disponible en más soportes, en más formatos y en más lenguajes, genera muchas preguntas y afecta a la elaboración y distribución de un periodismo de calidad. "La información ya no es exclusiva de los periodistas. Tenemos que conseguir un nivel cualitativo en todos los formatos que nos diferencie y nos haga útiles para el lector" (Vanessa Fransozi).

Las comunidades digitales asumen una capacidad como medios de difusión, pero no como 
canales a través de los cuales se descubran o den a conocer las denuncias. "El ocho por ciento de nuestro tráfico proviene de las redes", indica José María Irujo, "pero no las considero una herramienta para investigar". El factor más apreciado, especialmente por los periodistas free lance, es su facultad para generar una clara vocación comunitaria entre medio y audiencia: "Como periodista de El Mundo.es tenía 300 seguidores; pero ahora, gracias a mi blog, tengo casi 6.000. Eso sin Twitter hubiera sido imposible" (Eva Belmonte). Las redes, aseguran, han sido la única vía para generar confianza y darse a conocer:

El Diario.es ha sido capaz de crear una comunidad de lectores porque han sabido hacer llegar esa idea de que la calidad en la información y la autonomía del periódico dependen mucho de su audiencia, y de que si el producto que reciben es bueno, entonces se debe pagar por ello (Mar Cabra).

Aun así, se critica la superficialidad con la que las redes sociales afrontan el tratamiento informativo y la intromisión que ejercen en la privacidad de los usuarios. "Las comunidades permiten acercarnos más al lector pero a la vez nos llevan a crear una nueva agenda que limita el interés público. Es peligroso dejarse guiar por estas pautas. Es necesario seguir proporcionando a la audiencia discusiones diferentes" (Natália Levien Leal).

\section{El comportamiento de las audiencias}

La disposición de las audiencias respecto al pago por productos y servicios culturales en España vaticina una población que "no valora la importancia de la buena información y evita mayoritariamente el pago por ella" (Marcos García), pero que parece dispuesta a desembolsar pequeñas cantidades siempre y cuando los contenidos sean de entretenimiento y no estén disponibles en otro lugar de manera gratuita. "Me temo que a la audiencia de hoy le vale con un conglomerado de titulares que le da una idea básica de lo que está ocurriendo y poco más" (Esteban Urreiztieta).

El lector ha aceptado satisfactoriamente el coste del espectáculo televisivo pero no la idea de que una prensa independiente requiere de un apoyo económico que faculte su desarrollo. 'Fíjate en la lista de los libros más vendidos que publica Nielsen', lamenta Eric Frattini, "el ensayo más vendido de una semana puede llegar a los 7.000 ejemplares, pero si miras la novela, alcanza los 60.000". Diariamente, los periodistas tratan de hacer entender a los lectores que el conocimiento preciso de su cotidianeidad política y económica es imprescindible para asumir una posición fundamentada sobre las decisiones que les afectan.

Hace un mes puse al final de un artículo en mi blog la siguiente pregunta: ¿Cuánto crees que vale este post? Y el botón de PayPal para que la gente pudiera colaborar con, al menos, un euro. Bueno, el blog ha tenido más de 80.000 visitas desde entonces y nadie ha donado nada (Miguel Jara).

Además del comportamiento esquivo de la audiencia, se denuncia igualmente el 'malabarismo dialéctico' que algunos medios ponen en práctica para evitar decir que la información debe pagarse: "Hay medios cuyos contenidos digitales son gratuitos, pero que ofrecen camisetas con su logo para obtener ingresos. Y yo me pregunto, ipor qué pagar por una camiseta para leer un artículo, por qué no se cobra por el artículo en si?" (Pascual Serrano).

Algunos profesionales, sin embargo, creen que los lectores pagarán por un producto de calidad que se distinga de lo que todos ofertan, una vez entiendan esa distinción cualitativa, aunque admiten que falta mucha pedagogía entre grandes medios e instituciones afines para explicar por qué es necesario,

[...] ya que si los lectores siguen empeñados en recibir todo gratuitamente, a los periódicos solo les queda El Corte Inglés o el Estado (Pascual Serrano).

Nosotros pusimos en marcha una petición para recaudar 20.000 euros y poder realizar cuatro ediciones de Cafèambllet y, en 40 días, 1.619 personas aportaron 37.000 euros. Estas personas no recibieron ningún incentivo a cambio y es importante, porque no se aportó dinero para acceder a una información, sino para que otros pudieran acceder a ella. Y aquí ya no sé si estoy hablando de periodismo o de activismo (Albano Dante).

Los datos de la encuesta reflejan una situación similar en Brasil, donde los internautas, sobre todo los más jóvenes, están cada vez menos dispuestos a gastar en contenidos informativos. "Nuestra realidad es que los jóvenes se aferran a las redes sociales y se centran más en las relaciones que en el consumo de información para el desarrollo intelectual" (Silvana Araújo). Pese al 
avance en la cultura digital y el surgimiento de algunas experiencias innovadoras, "la venta de información periodística sigue siendo un desafio" (Benny Cohen).

Cuando se plantea la posibilidad del periodismo de pago, los profesionales brasileños recuerdan que seguimos proyectando una apreciación equivocada al creer que la producción periodística es perfectamente asumible y que nadie está dispuesto a pagar por el acceso a un contenido no diferenciado. Los lectores pagarían por la información de calidad si "[...] el mensaje online aportara valor propio, resultante de la credibilidad asumida por el canal y el profesional que firma el reportaje, y el texto impreso se ocupara de la carencia que supone la urgencia del consumo digital: el pensar" (Marcos Rolim).

La realidad es que solo una parte de la audiencia, aquellos que tengan intereses específicos, estarán dispuestos a realizar el desembolso: "el accidente de tráfico en mi calle puedo verlo gratis en Internet, así que pagaría por una información de profundidad, que resuelva mis dudas y me convenza de su veracidad" (Raquel Vieira).

\section{El reconocimiento del valor añadido}

La producción de información masiva y la falta de credibilidad de la comunicación libre alientan el consumo de las marcas más fiables y consolidadas. Es posible que, cuando se defina un modelo, los internautas reconozcan el valor añadido de la narrativa digital e "identifiquen la necesidad de dar contrapartida a la producción online, sea a través de la tolerancia a la publicidad o mediante la suscripción anual" (A.M.).

Algunos se arriesgan a decir que "el gran reto del periodismo digital es reinventarse en lo cotidiano y nutrirlo con las directrices de la versatilidad multimedia" (Natália Levien Leal). Desde otra perspectiva, hay quien asegura que

[...] nada se ha reinventado. Permanecen las premisas relevantes para la sociedad, los procesos de pauta, la creatividad, la palabra escrita en la pantalla, la organización de los contenidos y la necesidad de una práctica ética y comprometida con la búsqueda de la verdad (Nilson Vargas).

Desde los medios más pequeños hasta las cabeceras más conocidas, se percibe el interés y el esfuerzo conjunto para encontrar fórmulas de negocio oportunas que permitan la aceptación de los muros de pago. Sin embargo, a pesar del nicho de oportunidades que posibilita esta nueva coyuntura, muchos reporteros aplazan su entusiasmo y esperanza a la llegada "de un sistema de comunicación mejor" con tiempos y condiciones más estables.

Es nuestra intención en Diagonal pagar a los colaboradores, pero no podemos. Somos un medio pequeño, con una situación económica muy ajustada y unos fundamentos éticos por los que evitamos publicidad de ciertas empresas, así que los colaboradores y los suscriptores son nuestro mayor tesoro. Cuando nos llega el artículo de un colaborador ves el enorme esfuerzo que han hecho y sabes que en algunos casos su situación es muy precaria y que, aún así, le dedican tiempo a su tesón para a cambiar las cosas (María José Esteso).

El futuro del periodismo digital es incierto, pero el buen periodismo seguirá siendo necesario en cualquiera de sus formatos, aunque "aún no hemos encontrado el dispositivo ideal. Necesitamos más tiempo para saber si el periodismo digital será flexible, invisible, omnipresente o más o menos similar al papel" (Nilson Vargas).

\section{Conclusión}

El interés de la audiencia por el periodismo de calidad y la posibilidad de materializar esa sugestión en la compra de productos y contenidos on-line está presente en ambos países, aunque en Brasil se evidencia una predisposición ligeramente más positiva. Sin embargo, las respuestas rechazan nuestra hipótesis de partida, dado que gran parte de los lectores no están dispuestos a gastar en información mientras puedan encontrar de forma gratuita contenidos que satisfagan sus necesidades.

El actual panorama mediático demanda innovación, valor, eficiencia y velocidad en la manera de organizar y gestionar el producto, lo que impone a los medios digitales la incorporación en sus salas de redacción de nuevas prácticas, herramientas y aplicaciones en Red. En el caso español, aun con diferencias leves, todos los periodistas entrevistados mantienen una percepción 
positiva hacia los actuales modos de ideación, producción y difusión de contenidos informativos.

La experiencia de los periódicos brasileños parece encontrarse en un estado inicial, aún sin definir, que no garantiza la calidad mostrada en los diarios impresos. En todo caso, el 60\% de los expertos consultados observa que las empresas periodísticas, conscientes del potencial atractivo del ciberespacio, caminan hacia la construcción de un modelo innovador, volcando el esfuerzo en ofrecer al lector recursos que acentúen la divergencia y atiendan a sus intereses específicos.

En cuanto a las redes sociales, todos los profesionales españoles reconocen su capacidad como medios de difusión, pero no como canales a través de los cuales se posibilite la lectura de una información de calidad susceptible de ser vendida. En Brasil, las plataformas sociales también se constituyen como un elemento importante en el proceso de producción y publicación de los mensajes, aunque un 40\% de los periodistas señala la necesidad de seguir mostrando al lector que la experiencia cibersocial no sustituye el periodismo en profundidad.

Tanto en España como en Brasil, la percepción predominante es que solamente las personas que tengan intereses específicos pagarán por información de calidad. Un 50\% de los entrevistados estima que la inversión en el pago de contenidos ocurrirá cuando la audiencia logre entender la diferencia entre la comunicación libre, el valor añadido y la información diaria convencional.

Respecto al futuro del periodismo online, los españoles encuestados alertan de la necesidad de encontrar una forma para impugnar la insostenibilidad del sistema actual que mantiene a la audiencia acostumbrada a la gratuidad de la información. Para los expertos brasileños, la clave del éxito está en convencer a la audiencia de que el periodismo de calidad es posible y que seguirá ocupando su debido espacio independientemente del soporte que se utilice.

Ante todo, no podemos olvidar la precariedad en el acceso a los recursos digitales que afrontan las poblaciones más pobres y la importancia de reeducar a las personas que se resisten ante la cultura no presencial.

\section{Colaboración}

E.F. RODRÍGUEZ y C. FAVARETTO LOUZADA han contribuido en la concepción y diseño del estudio, análisis de datos y redacción final.

\section{Referencias}

AMERICAN PRESS INSTITUTE. How millennials get news: Inside the habits of America's first digital generation. 2015. Available from: <http://www.mediainsight.org/Pages/how-millennialsget-news-inside-the-habits-of-americas-first-digitalgeneration.aspx>. Cited: Sept. 29, 2015.

ASOCIACIÓN DE EDITORES DE DIARIOS ESPAÑOLES. LibrO blanco de la prensa Diaria. Madrid: Asociación de Editores de Diarios Españoles, 2014.

ASOCIACIÓN PARA LA INVESTIGACIÓN DE MEDIOS DE COMUNICACIÓN. Navegantes en la red: XV encuesta a usuarios de internet. 2012. Disponible en: <http://www.aimc.es/Navegantes-en-la-Red-.html>. Acceso: 29 sept. 2015.

ASOCIACIÓN PARA LA INVESTIGACIÓN DE MEDIOS DE COMUNICACIÓN. Navegantes en la red: XV encuesta a usuarios de internet. 2013. Disponible en: <http://www.aimc.es/Navegantes-en-la-Red-.html>. Acceso: 29 sept. 2015.

ASOCIACIÓN PARA LA INVESTIGACIÓN DE MEDIOS DE COMUNICACIÓN. Navegantes en la red: XV encuesta a usuarios de internet. 2014. Disponible en: <http://www.aimc.es/Navegantes-en-la-Red-.html>. Acceso: 29 sept. 2015.

BAHÓN, F. Muros de pago y oportunidades digitales. Cuadernos de Periodistas, 28 mayo 2014. Disponible en: <http://www.cuadernosdeperiodistas.com/muros-de-pagoy-oportunidades-digitales/>. Acceso: 29 sept. 2015.

BERGER, J.; MILKMAN, K. L. What makes online content viral? Journal of Marketing Research, v. 49, n. 2, p. 192-205, 2012. Available from: <http://papers.ssrn.com/sol3/papers. cfm?abstract_id=1528077>. Cited: Sept. 29, 2015.

BRADER, T. Campaigning for hearts and minds. Chicago: University of Chicago Press, 2006.

BROCK, G. Out of print: Newspapers, journalism and the business of news in the digital age. London: Kogan Page, 2013.

BRUNO, N.; NIELSEN, R. K. Survival is success. Journalistic online start-ups in Western Europe. Oxford: University of Oxford, 2012. Available from: <http://reutersinstitute.politics.ox.ac.uk/ publication/survival-success>. Cited: Sept. 29, 2015. 
CHYl, H. I. Paying for what? How much? And why (not)? Predictors of paying intent for multiplatform newspapers. International Journal on Media Management, v. 14, n. 3, p. 227-250, 2012. Available from: <http://www.tandfonline.com/ doi/full/10.1080/14241277.2012.657284\#.VHW518m9bw8>. Cited: Apr. 12, 2013

CHYI, H. I.; LEE, A. M. Theorizing online news consumption: A structural model linking preference, use, and paying intent. International Symposium on Online Journalism, n. 3, p. 20-21, 2012a. Available from: <https://online.journalism.utexas. edu/2012/papers/chyiandlee.pdf>. Cited: Sept. 29, 2015.

$\mathrm{CHYI}, \mathrm{H}$. I.; LEE, A. M. Will the Internet disrupt? A reality check on format preference for traditional and digital content across five media. WORLD MEDIA ECONOMICS \& MANAGEMENT CONFERENCE, 10., Thessaloniki, Greece, 2012b. Annales.. Thessaloniki, Greece: University of Texas at Austin, 2012b. Available from: <http://www.academia.edu/3448049/ Will_the_Internet_Disrupt_A_Reality_Check_on_the_ Performance_of_Online_and_Traditional_Formats_Across_ Five_Media>. Cited: Jan. 4, 2014.

DE LA TORRE, L.; TÉRAMO, M. T. La calidad de la información periodística: estrategias para su observación. Coincidencias y divergencias entre los medios y el público. Estudios sobre el Mensaje Periodístico, v. 21, p. 135-144, 2015. Edición especial. http://dx.doi.org/10.5209/rev_ESMP.2015.v21.50666

DI GIACOMO, F. O conteúdo grátis está transformando o jornalismo num grande anúncio de refrigerantes? Revista Super Interessante, São Paulo, 6 set. 2013. Disponível em: <http://super.abril.com.br/blogs/newsgames/o-conteudogratis-esta-transformando-o-jornalismo-num-grandeanuncio-de-refrigerantes/>. Acesso em: 27 out. 2015.

EDMONDS, R. Is paid online content a solution or impossible dream? St. Petersburg: Poynter, 2009. Available from: <http:// www.poynter.org/news/mediawire/94173/is-paid-onlinecontent-a-solution-or-impossible-dream/>. Cited: Sept. 29, 2015.

FONDEVILA, J. F. La prensa digital en España: ¿un negocio viable para emprendedores? In TURMO, F. S.; LASSA, J. J. V. (Coord.). El periodismo digital analizado desde la investigación procedente del ámbito académico. Zaragoza: Asociación de Periodistas de Aragón, 2012. Disponible en: $\quad$ <http://decimotercero.congresoperiodismo.com/pdf/ Libroelectronico2012.pdf>. Acceso: 29 sept. 2015.

GÓMEZ-MOMPART, J. L.; GUTIÉRREZ-LOZANO, J.F.; PALAUSAMPIO, D. La calidad periodística en España según la percepción de los periodistas. Estudios sobre el Mensaje Periodístico, v. 21, p. 13-30, 2015. Edición especial. http://dx.doi. org/10.5209/rev_ESMP.2015.v21.50647

GOYANES, M. The value of proximity: Examining the willingness to pay for online local news. International Journal of Communication, v. 9, p. 1505-1522, 2015. Available from: <http://ijoc.org/index.php/ijoc/article/view/3388 >. Cited: Sept. $1,2015$.

HAMILTON, J. T. All the news that's fit to sell. New Jersey: Princeton University Press, 2004.
HUNTER, M. L.; WASSENHOVE, L. Disruptive news technologies: Stakeholder media and the future of watchdog journalism business models. Fontainebleau: Insead, 2010. Available from: <http://papers.ssrn.com/sol3/papers.cfm?abstract_id=158 2324>. Cited: Sept. 29, 2015.

JARQUE, J.M.; ALMIRÓN, N. Periodismo para Internet o periodismo, y punto. Palabra Clave, v. 11, n. 2, 2008. Disponible en: <http://palabraclave.unisabana.edu.co/index.php/palabra clave/article/view/1426/1562>. Acceso: 2 ago. 2011.

JUSTEL, S. Transformación en la audiencia, transformación en los medios: un marco para el estudio de los retos y estrategias de las empresas periodísticas. AdComunica, n. 4, 2012. http:// dx.doi.org/ 10.6035/2174-0992.2012.4.3

KPMG International. Consumers and convergence III: Consumer taking charge. [S.I.]: KPMG International Cooperative, 2008. Available from: <http://www.kpmg.com/cn/en/ issuesandinsights/articlespublications/pages/takingcharge-0-200905.aspx>. Cited: Sept. 29, 2015.

KPMG International. Consumers and convergence IV: Convergence goes mainstream: Convenience edges out consumer concerns over privacy and security. 2010. Available from: <http://www.kpmg.com/cn/en/issues andinsights/articlespublications/pages/convergence-goesmainstream-o-201007.aspx>. Cited: Sept. 29, 2015.

KPMG International. Consumers and convergence $V$ : The converged lifestyle. 2011. Available from: <http://www.kpmg. $\mathrm{com} / \mathrm{mx} / \mathrm{es} /$ issuesandinsights/articlespublications/paginas/ the-converged-lifestyle.aspx>. Cited: Sept. 29, 2015.

LEWIS, C. Seeking new ways to nurture the capacity to report. Nieman Reports. v. 62, n. 1 p. 23-26, 2008. Available from: <http://www.nieman.harvard.edu/reportsitem.aspx?id= 100060>. Cited: Apr. 12, 2012.

LOBO, R. Ya no te miden por lo que vales, sino por lo que cuestas. España: Jotdown, 2012. Disponible en: <http://www.jotdown. es/2012/11/ramon-lobo-ya-no-te-miden-por-lo-que-valessino-por-lo-que-cuestas/>. Acceso: 13 feb. 2013.

MARSHALL, L. A. Sociedade da hipercomunicação. São Paulo: Observatorio de Empresa, 2014. Disponível em: <http:// portalintercom.org.br/anais/centrooeste2013/resumos/R360301-1.pdf>. Acesso em: 27 out. 2015.

MARTÍN, A. (Coord.). Seguiremos informando. Madrid: Los Libros de la Catarata, 2010.

MCCHESNEY, R.; PICKARD, V. (Ed.). Will the last reporter please turn out the lights: The collapse of journalism and what can be done to fix it. New York: The New Press, 2011.

MITCHELL, A. The state of the news media: An annual report on American journalism. Washington, DC: Pew Research Center, 2014. Available from: <http://www.journalism.org/ 2015/04/29/state-of-the-news-media-2015/>. Cited: Sept. 29,2015

MITCHELL, A.; PAGE, D. The state of the news media: An annual report on American journalism. Washington, DC: Pew Research Center, 2015. Available from: <http://www. journalism.org/packages/state-of-the-news-media-2014/>. Cited: Sept. 29, 2015. 
MITCHELL, A.; ROSENSTIEL, T. The state of the news media: An annual report on American journalism. Washington, DC: Pew Research Center, 2013. Available from: <http://www. stateofthemedia.org/2013/>. Cited: Sept. 29, 2015.

NELSON, A. Continental shift: New trends in private U.S. funding for media development. Washington, D.C.: Center for International Media Assistance (CIMA) \& National Endowment for Democracy (NED), 2011. Available from: <http://www. cima.ned.org/resource/continental-shift-new-trends-inprivate-u-s-funding-for-media-development/>. Cited: Sept. 29, 2015.

NEWMAN, N.; LEVY, D. A. Reuters Institute Digital News Report 2013: Tracking the future of news. Oxford: Reuters Institute for the Study of Journalism, 2013. Available from: <http://www. digitalnewsreport.org/survey/2013/>. Cited: Sept. 29, 2015.

NEWMAN, N.; LEVY, D. A. Reuters Institute Digital News Report 2014: Tracking the future of news. Oxford: Reuters Institute for the Study of Journalism, 2014. Available from: <http://www. digitalnewsreport.org/survey/2014/>. Cited: Sept. 29, 2015.

NEWMAN, N.; LEVY, D. A.; NIELSEN, R. K. Reuters Institute Digital News Report 2015: Tracking the future of news. Oxford: Reuters Institute for the Study of Journalism, 2015. Available from: <http://www.digitalnewsreport.org>. Cited: Sept. 29, 2015.

ORIELLA PR NETWORK. The new normal for news: Have global media changed forever? Canbera: Benton Foudation, 2011-2013. Available from: <http://www2.horngroup.com/assets/Uploads /Oriella-Assets/Brands2LifeODJSv4.pdf>. Cited: Sept. 29, 2015.

PALACIO, L. (Dir.). Informe anual de la profesión periodística. Madrid: Asociación de la Prensa de Madrid, 2014.

PALACIO, L. (Dir.). Informe anual de la profesión periodística. Madrid: Asociación de la Prensa de Madrid, 2013.

RAMÍREZ DE LA PISCINA, T. et al. Periodismo de calidad en tiempos de crisis: un análisis de la evolución de la prensa europea de referencia (2001-2012). Revista Latina de Comunicación Social, v. 69, p. 248-274, 2014. Disponible en: <http://www.revistalatinacs.org/069/paper/1011 UPV/14t. html>. Acceso: 29 sept. 2015

REQUEJO-ALEMÁN, J. L.; LUGO-OCANDO, J. Assessing the sustainability of Latin American investigative non-profit journalism. Journalism Studies, v. 15, n. 5, p. 522-532, 2014. http://dx.doi.org/10.1080/1461670X.2014.885269

RIVERO, M. Quien paga manda. Periodistas, n. 34, p. 16-19, 2014. Disponible: <http://fape.es/file/4731_Periodistas\%20 34.pdf>. Acceso: 12 mayo 2015.

SABA, J. No more free ride? Fountain Valley: Editor \& Publisher, 2011. Available from: <http://editorandpublisher.com/ Article/No-More-Free-Ride->. Cited: Sept. 29, 2015.

SALAVERRÍA, R. Extrema turbulencia en los medios en 2011 Anuario ThinkEPI, v. 6, p. 161-165, 2012. Disponible en: <http:// dadun.unav.edu/handle/10171/21940>. Acceso: 29 sept. 2015.

SHIELDS, M. Web publishers divided over the value of charging. Fountain Valley: Editor \& Publisher, 2009. Available from: <http://editorandpublisher.com/PrintArticle/WebPublishers-Divided-Over-the-Value-of-Charging > . Cited: Sept. 29, 2015

SOENGAS-PÉREZ,X. El pluralismo y el control de la información en las televisiones generalistas españolas. Estudios sobre el Mensaje Periodístico, v. 21, p. 199-207, 2015.Edición especial. http://dx.doi.org/10.5209/rev_ESMP.2015.v21.50675

TRANSPARENCY INTERNATIONAL. Puttig corruption out of business. 2012. Available from: <http://www.transparency. org/news/feature/putting_corruption_out_of_business $>$. Cited: Sept. 29, 2015.

VALLÉS, M. S. Entrevistas cualitativas. Madrid: Centro de Investigaciones Sociológicas, 2002.

VARA-MIGUEL, A.; SANJURJO, E.; DÍAZ-ESPINA, C. Paid news vs. free news: Evolution of the WSJ.com business model from a content perspective (2010-2012). Communication \& Society, v. 27, n. 2, p. 147-167, 2014. Available from: <http://www. unav.es/fcom/communication-society/es/articulo.php?art_ $i d=494$ > . Cited: Sept. 29, 2015. 